\title{
Les effets tératogènes du tabac sur les structures oro-faciales des souris
}

\section{RÉSUMÉ}

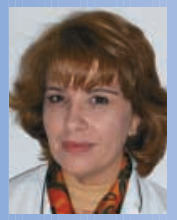

Souha BENYOUSSEF BOUDEGGA Professeur en médecine et chirurgie buccales, Unité de chirurgie buccale,

CHU Farhat Hached Sousse, Tunisie.

\section{Badreddine SRIHA}

Maïtre de Conférence

agrégé d'anatomie

et de cytologie pathologique

CHU Farhat Hached Sousse, Tunisie

\section{Lamia OUALHA}

Maïtre de Conférence

agrégée en médecine et chirurgie buccales

Clinique dentaire Monastir, Tunisie.

\section{Aicha ZAGHBANI}

Assistante hospitalo-universitaire en médecine et chirurgie buccales, CHU Farhat Hached Sousse, Tunisie.

\section{Fredj BENYOUSSEF}

Chef de service de pharmacie,

CHU Farhat Hached Sousse, Tunisie.

\section{Chadly BACCOUCHE}

Chef de service de médecine dentaire,

CHU Farhat Hached Sousse, Tunisie.

Les auteurs rapportent une étude réalisée sur des embryons de souris exposées aux différentes phases du tabac durant toute leur période de gestation. Les résultats des coupes histologiques réalisées étaient alarmants et signifiants, affirmant l'effet tératogène du tabac et la présence d'anomalies de développement concernant les maxillaires et la face chez les embryons des souris exposées.

\section{- tératologie}

- tabagisme

- malformations maxillo-faciales
AOS 2008:243:249-263

DOI:10.1051/AOS:2008031

(C) AEOS / EDP Sciences 


\section{Introduction}

$>$

Les tendances actuelles attribuent au tabac un effet tératogène sur l'embryon humain, outre son rôle dans la genèse de tumeurs et son rôle causal dans plusieurs maladies surtout cardiaques et respiratoires, cependant aucun syndrome spécifique lié au tabac n'a été décrit. Plusieurs études expérimentales sont faites à ce propos, les démarches sont différentes, les résultats sont modestes n'aboutissant pas à des conclusions satisfaisantes.

Ainsi notre travail s'intègre dans ce cadre. Notre objectif est d'établir le rôle du tabac dans la genèse des malformations maxillofaciales d'autant plus qu'il y $a$, d'après les données bibliographiques, une association significativement positive entre l'exposition au tabac et le risque de fente orale. Par ailleurs, les malformations qui touchent le fœtus humain sont très fréquentes. Mais, si on compare la fréquence de ces phénomènes avec les animaux d'expérimentation (souris), on trouve que les malformations chez les animaux sont de loin moins fréquentes que chez I'homme.

\section{Matériels et méthodes}

Nous nous proposons de réaliser une étude sur les maxillaires et la face des embryons de souris exposées aux différentes phases du tabac durant toute leur période de gestation.

En effet, il suffit par exemple de comparer la durée des deux grossesses : chez l'homme, elle est de l'ordre de 280 jours, alors qu'elle est de l'ordre de 21 jours chez les souris.

Bien que l'écart temporel soit considérable, cela n'empêche pas, toutefois, que l'on puisse procéder à une comparaison des phases d'évolution du développement embryonnaire, à savoir la phase de morphogenèse corporelle, la phase embryonnaire et la phase fotale que connaissent aussi bien les fœtus humains que les fœtus animaux.

- Pendant la phase de morphogenèse corporelle, la cellule œuf passe au stade de 2 cellules, ensuite de 4 cellules, puis de 16 cellules, pour avoir finalement la forme d'une sphère ressemblant à une mûre: c'est le stade morula. Vers le $4^{e}$ jour, un liquide venant de la cavité utérine s'infiltre dans la morula et occupe des espaces intercellulaires; ces espaces fusionnent et forment une cavité unique et large : c'est le blastocèle et on est alors dans le stade de blastocyste. Celui-ci s'implante dans l'endomètre maternel où il subit des modifications morphologiques formant un disque embryonnaire didermique composé d'endoderme embryonnaire et d'épiblaste (futur ectoderme et mésoderme). Chez les vertébrés, les étapes de morphogenèse corporelle sont similaires mais la durée diffère : elle dure 2 semaines chez l'homme et $\mathbf{1 1}$ jours chez la souris.

- Pendant la phase embryonnaire, les trois couches germinales vont donner naissance à des tissus et organes spécifiques: c'est la période de l'organogenèse, durant laquelle les principales malformations congénitales peuvent survenir à la suite d'une exposition 
de l'embryon aux agents tératogènes. Chez les mammifères, les étapes de la phase embryonnaire sont très comparables. En effet, l'embryon humain et l'embryon animal sont tellement similaires qu'il est difficile de les distinguer. Cependant cette phase dure 3 jours chez la souris alors que sa durée chez I'homme est de l'ordre de 6 semaines.

- Pendant la phase fœtale, la forme du foetus devient clairement perceptible : cette phase dure 8 jours chez la souris et 6 mois chez I'homme.

Par ailleurs, si nous comparons le développement embryonnaire chez les deux sujets (les animaux de laboratoire et l'homme), nous remarquons une grande similitude.

Ainsi on est quasi certain que lorsqu'il y a malformation chez la souris au cours d'une phase quelconque du développement à cause d'un agent tératogène, elle se serait également produite chez l'homme, pendant la même phase, sous l'influence du même stimulus.

\section{Matériels}

Pour réaliser l'expérience nous avons utilisé quatre couples de souris blanches d'une race spécifique qui se multiplient d'une façon isolée depuis plus de 10 ans. Les souris témoins ont évolué de façon normale dans des conditions de laboratoire.

L'âge : I'âge moyen de croissance des souris de l'expérience (fig. 1) est entre 3 mois et 4 mois.

Les feuilles de tabac sont mélangées avec l'eau donnée aux souris. Les composants de la phase particulaire du tabac tels que la nicotine, le cadmium, le plomb, la cyanide se trouvent ainsi dissous dans l'eau qui devient de couleur marron, les feuilles de tabac sont aussi mélangées avec l'alimentation.

En outre, les souris sont exposées à la fumée du tabac (la phase gazeuse: $\mathrm{CO}, \mathrm{CO} 2$ ) d'une façon aléatoire. Les souris étaient sous l'influence des différentes phases du tabac durant toute la période de gestation (20 - 21 jours).

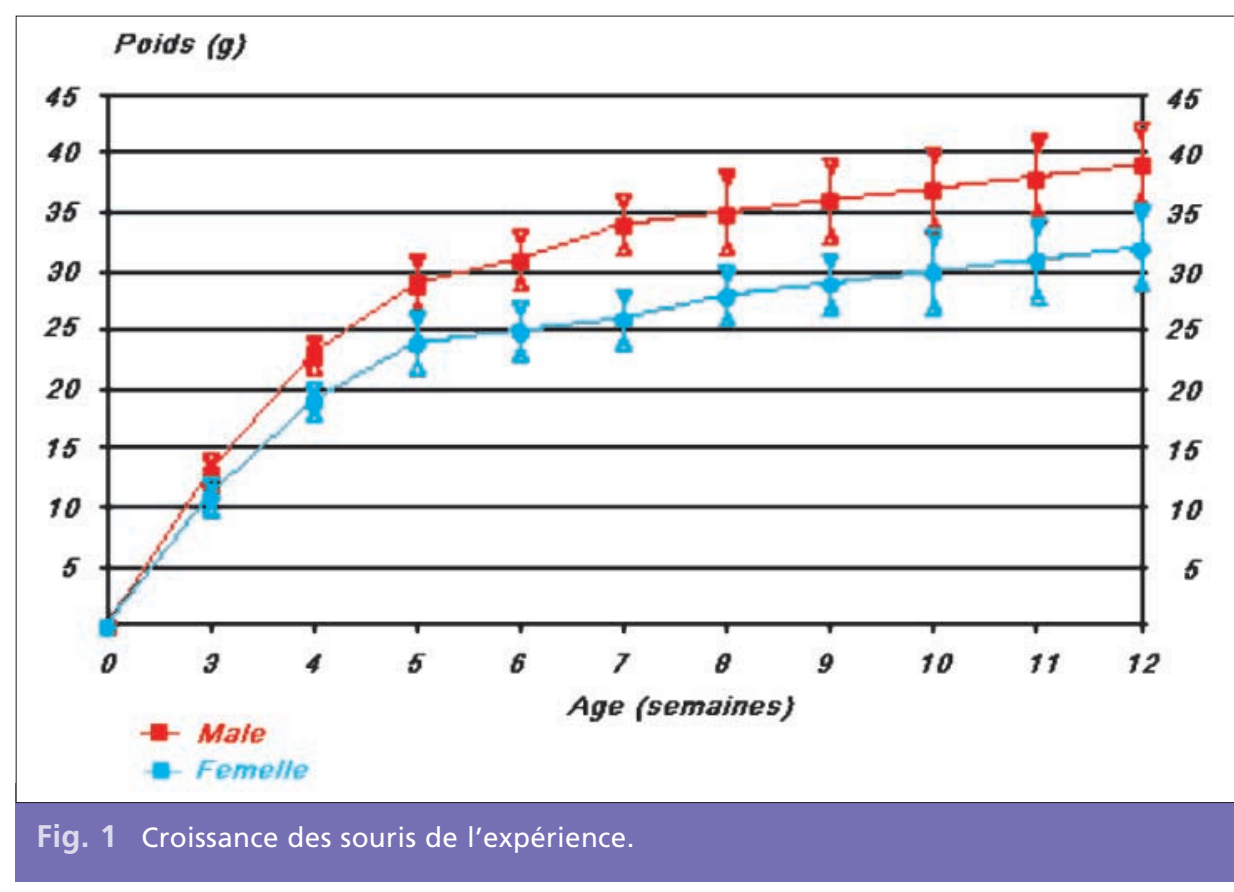



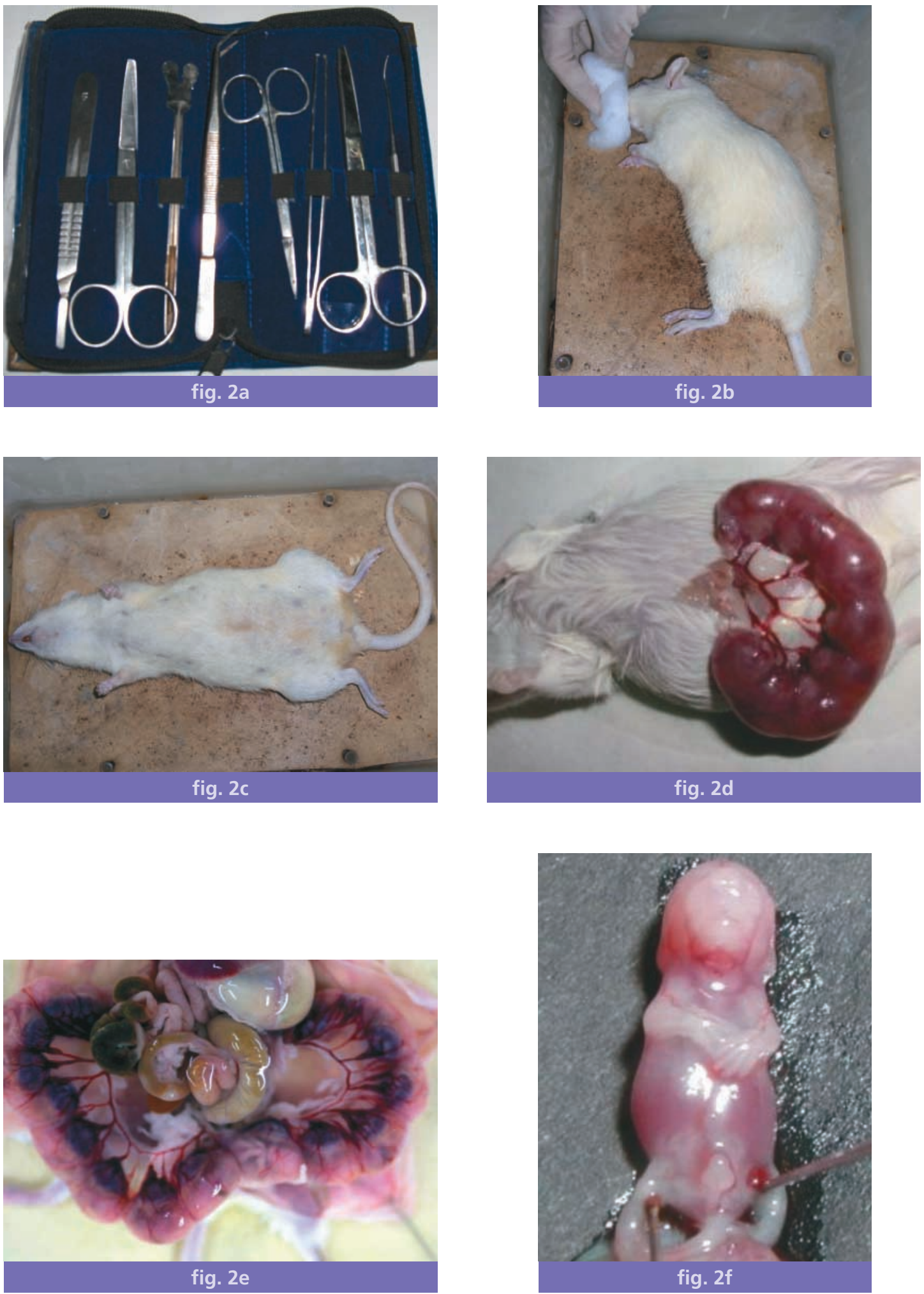

Fig. 2 a à $f$ Dissection de la souris au $20^{\mathrm{e}}$ jour de la gestation.

252 


\section{Méthodes}

Pour avoir des résultats statistiquement valables, nous avons colligé au moins 33 souris gestantes.

$\mathrm{Au} 20^{\mathrm{e}}$ jour de la gestation, nous sacrifions tout d'abord la souris par transduction spinale ensuite nous réalisons une dissection.

Après avoir cherché l'utérus et les fœtus, nous coupons le placenta et enfin nous fixons les fœtus dans le formol ou l'alcool (fig. 2 a à f).

Par la suite, les fœtus sont transmis au service $\mathrm{d}$ 'anatomie pathologique où ils sont examinés macroscopiquement en se concentrant sur l'état des deux mâchoires afin d'observer une malformation. La taille et le diamètre de chaque fœtus sont respectivement notés.

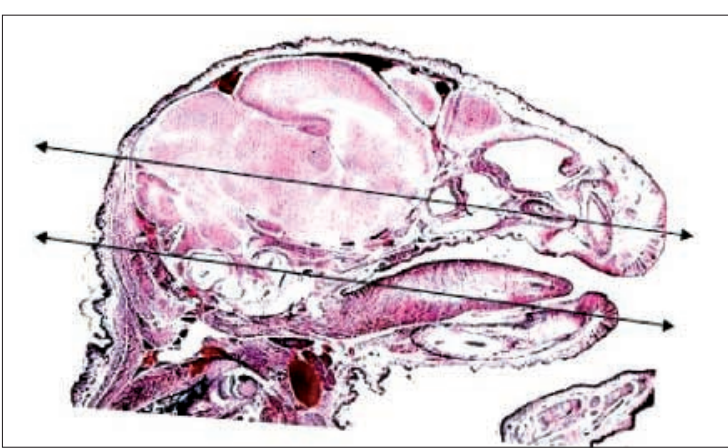

Fig. 3 Coupe histologique sagittale médiane de la tête d'un embryon de souris illustrant les niveaux de coupes effectués.

\section{Résultats}

Notre échantillon comprend 41 lames :

- 33 lames correspondent aux coupes des mâchoires des souris exposées au tabac ;

- 8 lames correspondent aux coupes des mâchoires des souris témoins.
Nous réalisons des coupes au niveau de la mâchoire supérieure passant par le tragus et un point sous-nasal (fig. 3). Les coupes sont enrobées puis colorées à l'hématoxyline et l'éosine afin de les observer au microscope et de les examiner histologiquement selon deux grossissements : 25 et 40 .

D'autre part, nous avons réalisé 8 coupes à partir de 8 fœtus provenant de souris témoins qui ont évolué de façon normale dans des conditions de laboratoire afin de pouvoir assurer une comparaison à l'échelle microscopique entre les mâchoires des fœtus de l'expérience et des fœtus témoins.

Toutes les coupes réalisées sont des coupes transversales (fig. 4).

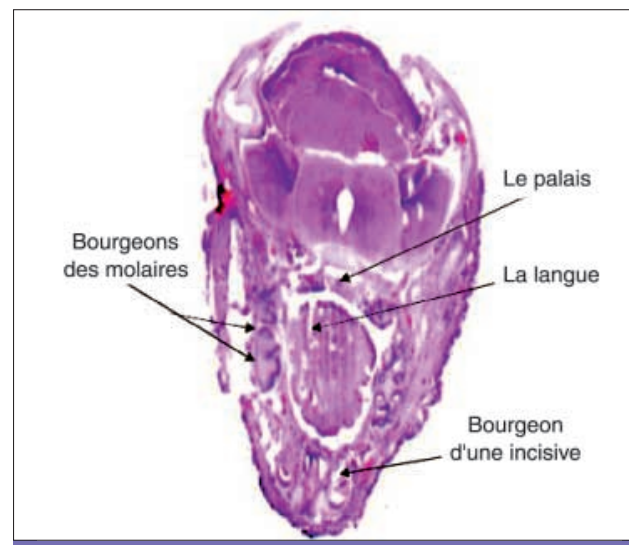

Fig. 4 Coupe transversale passant par la mâchoire supérieure d'un embryon de souris.
Depuis la lecture des premières lames, nous nous sommes rendu compte de la présence de malformations sur les structures oro-faciales des souris ayant été exposées au tabac (tableau I). 


\section{Tableau I}

Tableau illustrant les différentes malformations des structures oro-faciales observées chez les 33 fœtus de souris gestantes exposées au tabac.

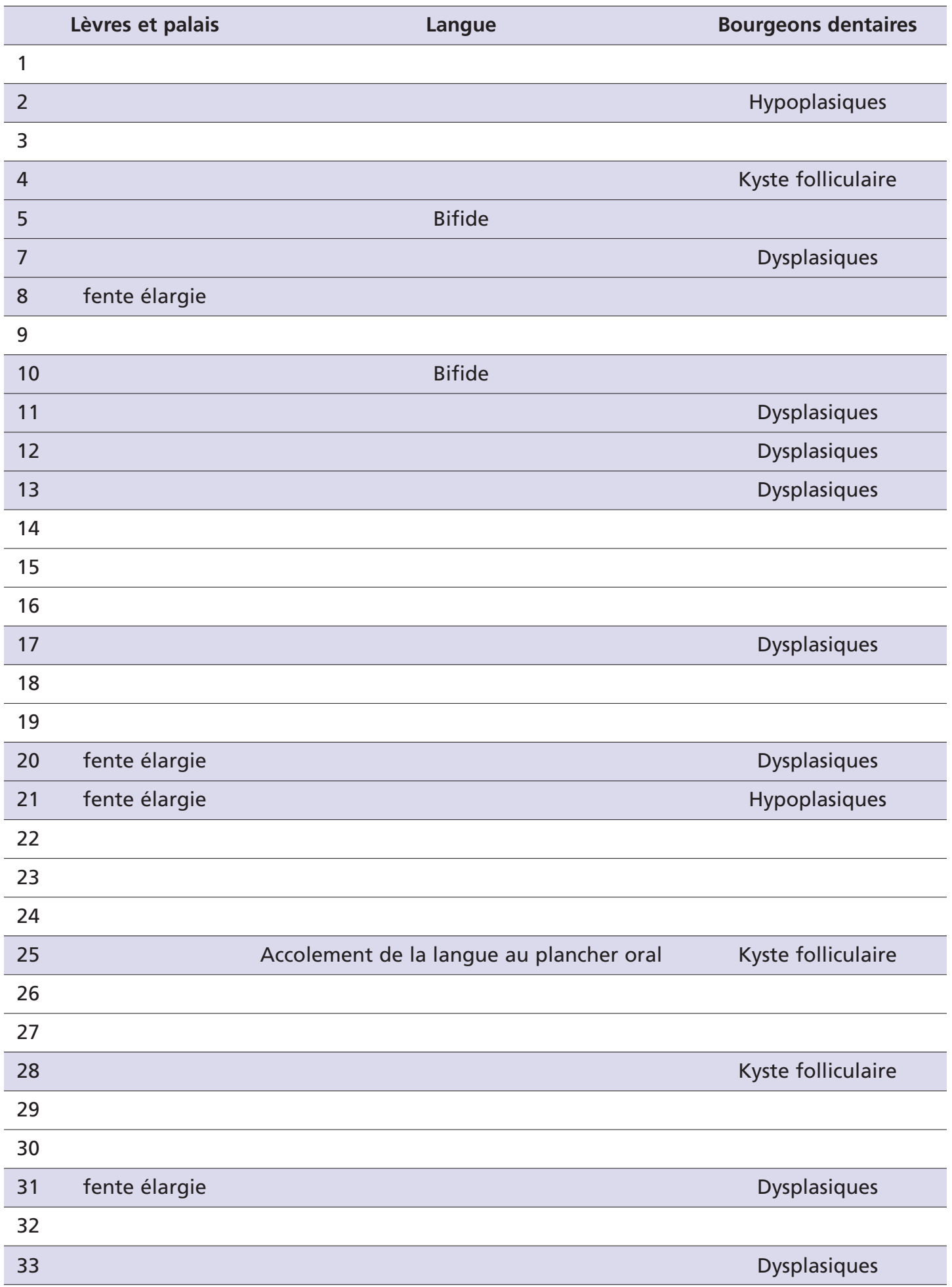


Les résultats de nos expériences ont montré une fréquence de malformations de l'ordre de $48 \%$ soit 16 fœtus sur les 33 exposés.

L'ordre de fréquence des types de malformations est le suivant :

-8 bourgeons dentaires dysplasiques (fig. 5,6 ) : - 3 kystes folliculaires (fig. 7) ;

-2 bourgeons dentaires hypoplasiques (fig. 8);

-4 fentes labiales palatines très élargies (fig. 9, 10) ;
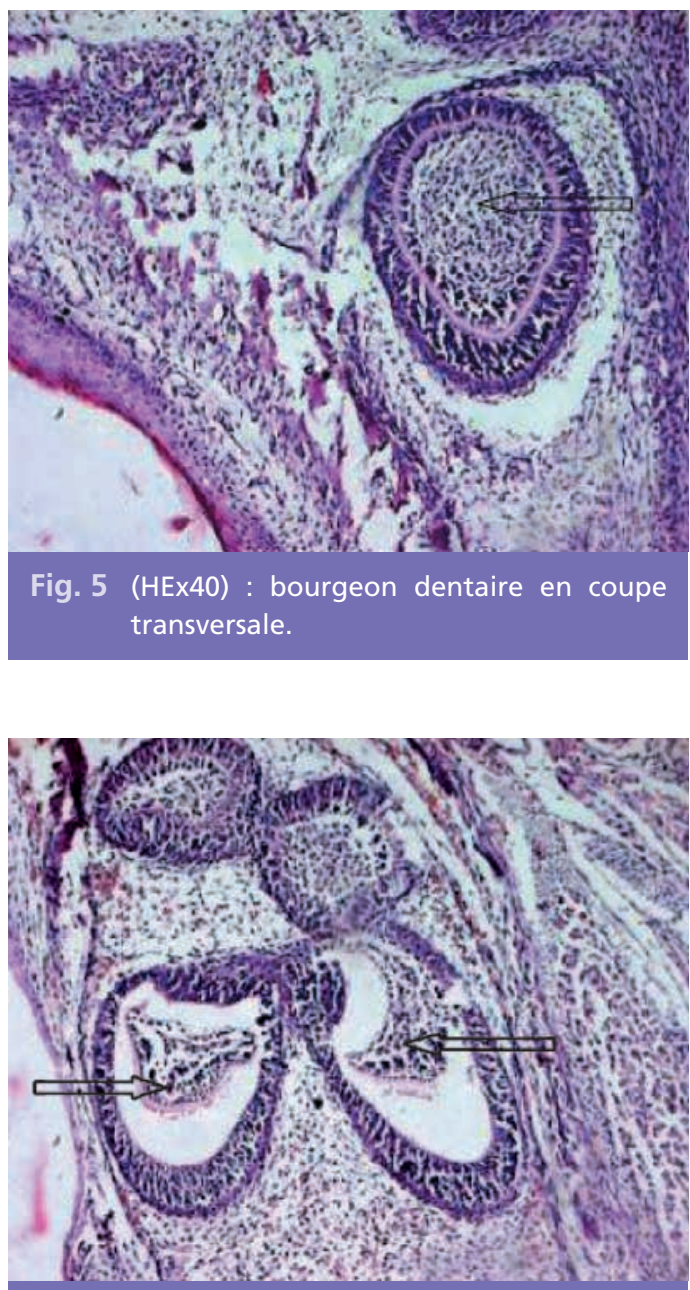

Fig. 7 (HEx40) : kyste folliculaire.
-2 langues bifides (fig. 11) ;

- 1 accolement de la langue au plancher buccal

(fig. 12, 13, 14).

4 fœtus sur 33 présentaient en même temps deux malformations.

Cependant aucune fente palatine isolée n'a été observée (fig. 15).

Aucune malformation n'a été détectée au niveau de l'échantillon des embryons témoins.

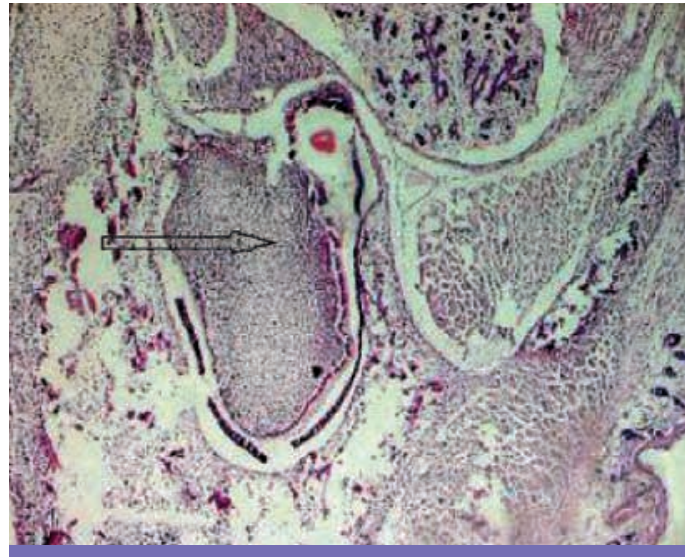

Fig. 6 (HEx40) : bourgeon dysplasique.

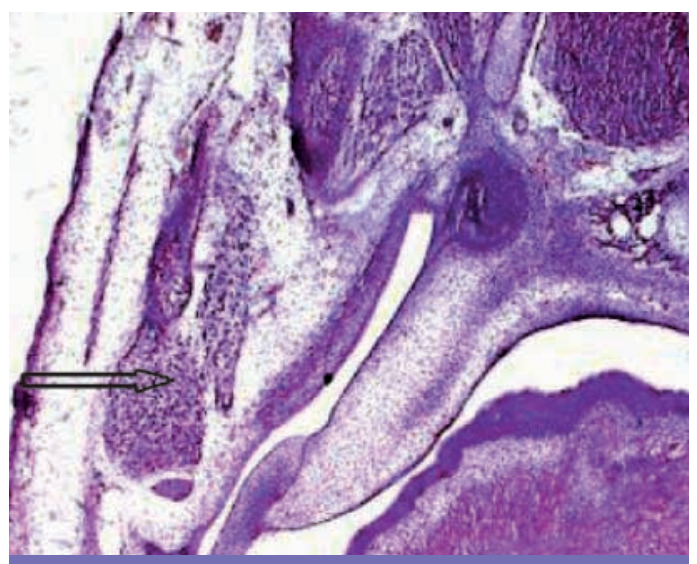

Fig. 8 (HEx40) : bourgeon hypoplasique. 


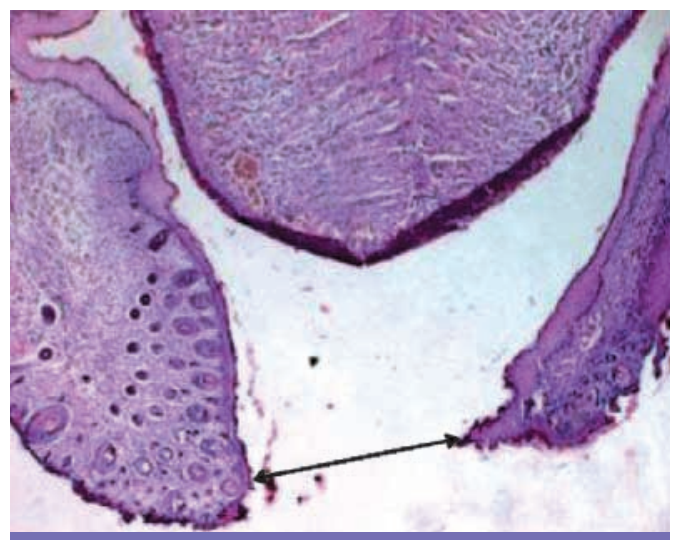

Fig. 9 (HEx40) : fente labiale supérieure.
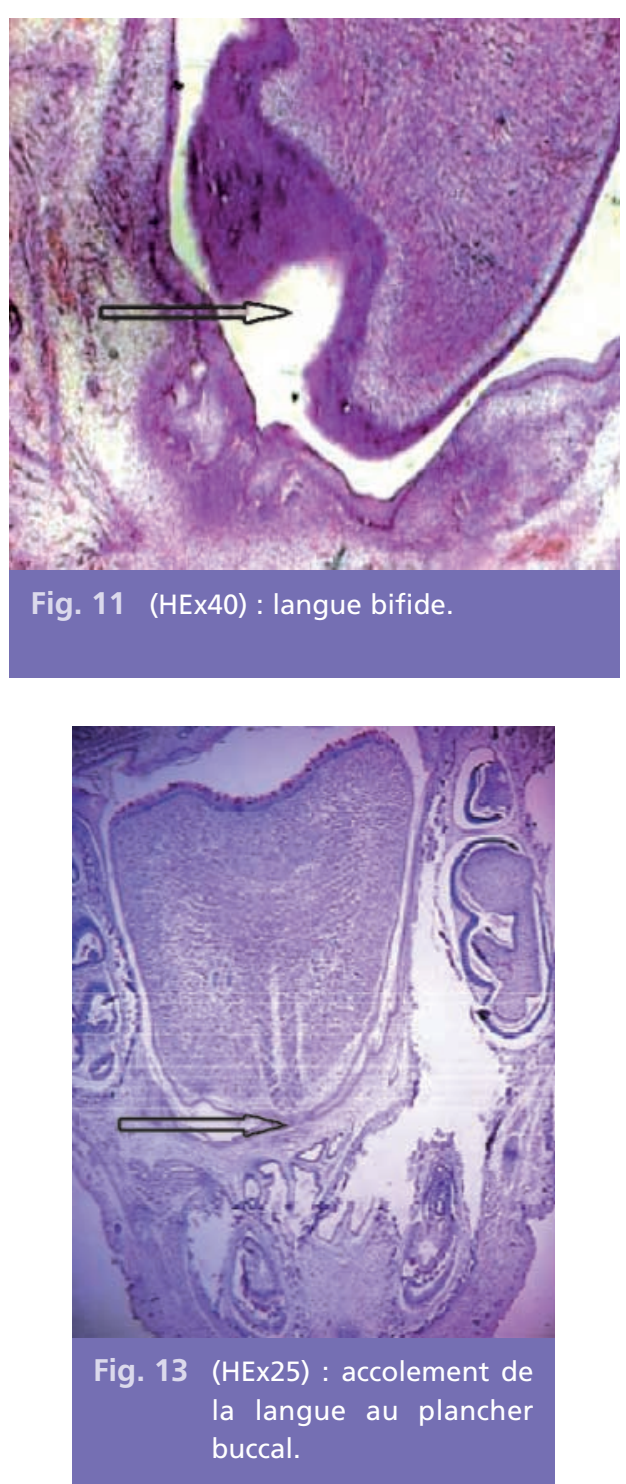

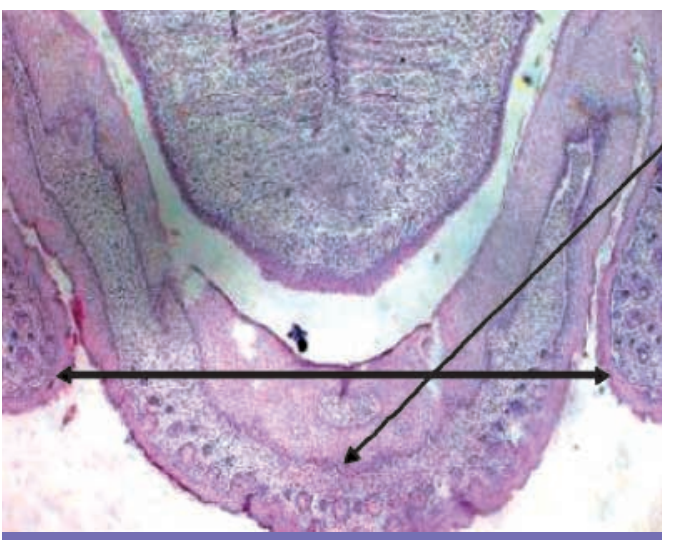

Fig. 10 (HEx40) : fente labiale supérieure très élargie.

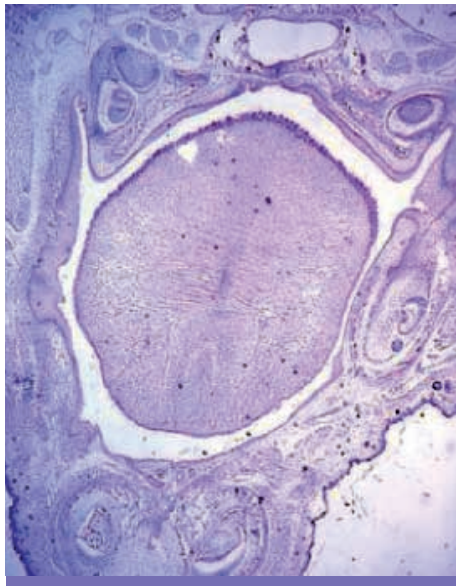

Fig. 12 (HEx25) : aspect d'une langue normale.

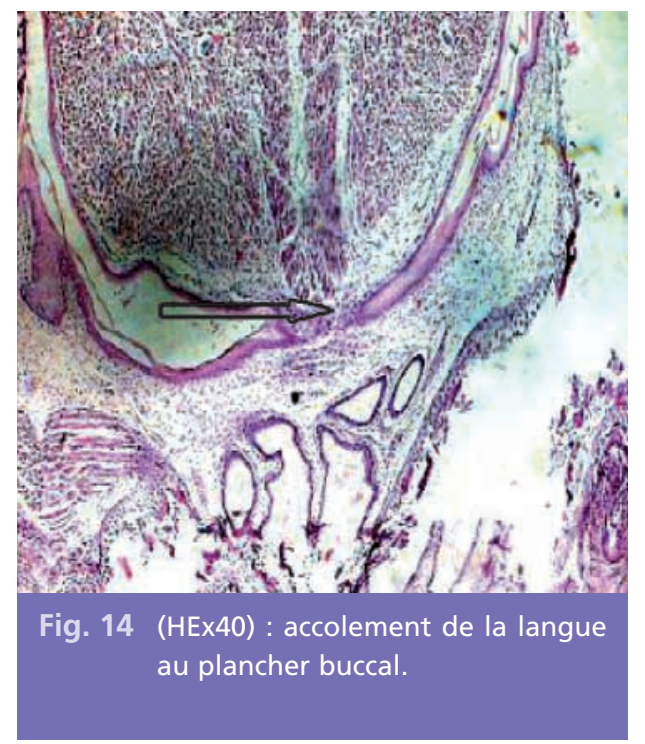




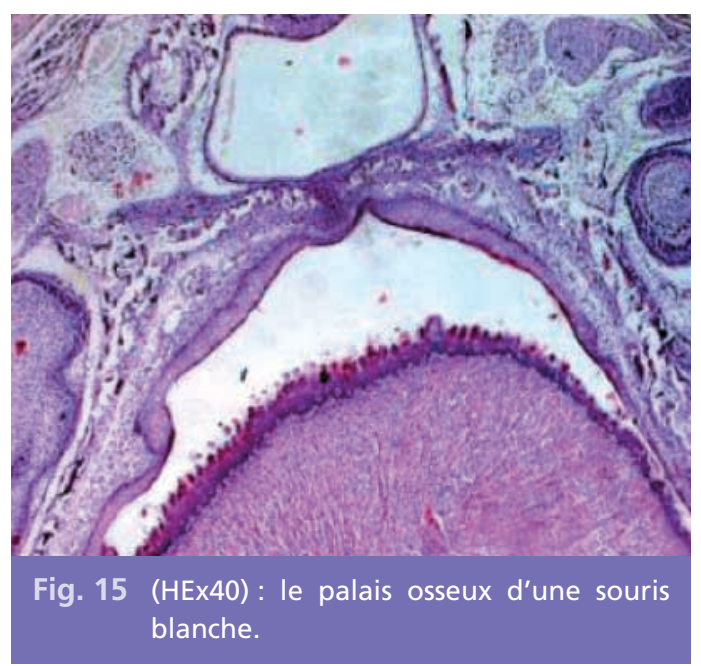

\section{Discussion}

Bien que l'exploitation des souris reste l'un des meilleurs moyens d'expérimentation pour I'homme, l'auto-évaluation de notre étude nous permet de soulever plusieurs points critiques:

1. comment déterminer la dose et la forme de tabac à effet tératogène pour les souris d'autant plus que nous avons utilisé pour notre expérience le tabac sous trois formes (dilué à l'eau, mélangé à la nourriture et en fumée) ?

2. en sachant que les composants du tabac sont divers et variés, comment déterminer celui qui est à l'origine des malformations ?

3. quelle est la période de sensibilité tératogénique au tabac?

4. quel est le mécanisme qui induit des malformations orofaciales?

5. existe-t-il d'autres malformations intéressant le reste du corps ?

6. existe-t-il des gènes prédisposant à l'apparition de ces malformations ?
7. comment estimer l'influence d'autres facteurs non pris en considération dans notre étude à savoir l'association du tabac à d'autres agents de pollution de l'environnement ?

8. peut-on extrapoler ces résultats à la race humaine?

Pour répondre à ces questions, il faut rappeler[4] qu'il existe trois périodes de sensibilité aux agents tératogènes dans l'espèce humaine :

1. Une période dite d'insensibilité tératogénique qui correspond à la période de la vie libre de l'embryon. Durant cette période, l'embryon peut être tué. Sinon aucune anomalie ne peut être déterminée durant cette période, exception faite de mosaïques chromosomiques (cas théorique d'un agent tératogène interférant avec la dynamique du fuseau mitotique).

Au cours de nos expériences, nous n'avons détecté aucune rupture de la gestation; ceci peut être expliqué par sa courte durée chez les souris. 
2. Une période de grande sensibilité tératogénique qui correspond à la période embryonnaire du $15^{\mathrm{e}}$ jour à la fin de la $8^{\mathrm{e}}$ semaine de gestation.

Durant cette période, les facteurs tératogènes déterminent des anomalies graves (embryopathies) qui sont souvent létales, ou au moins très visibles et/ou invalidantes.

En règle générale, les anomalies sont d'autant plus graves qu'elles sont plus précoces.

Un agent tératogène affecte souvent, simultanément, le développement de plusieurs organes ayant la même période de sensibilité. En d'autres termes, la période d'exposition à un agent tératogène est parfois plus importante que sa nature dans la genèse d'une association donnée d'anomalies (syndrome malformatif).

Jusqu'à maintenant, on n'attribue au tabac aucun syndrome malformatif. Cependant, on incrimine une association significativement positive entre l'exposition au tabac et le risque de fente orale.

Il existe cependant de nombreux agents tératogènes dont les actions sont spécifiques, soit parce qu'ils agissent en se liant à des récepteurs, soit parce qu'ils s'accumulent dans des structures spécifiques. Ceci dit, on n'a pas identifié encore au tabac un récepteur spécifique $[10,14]$, récepteur qui a été décrit, par exemple, dans les cas suivants :

- les androgènes qui n'ont d'action que sur les organes génitaux externes et internes qui sont leurs organes cibles normaux (seuls ces organes expriment le récepteur des androgènes) ;

- l'iode et les anti-thyroïdiens de synthèse qui n'agissent que sur la thyroïde fœtale ;

- les psychotropes qui s'accumulent dans le Système Nerveux Central et perturbent son développement de façon préférentielle.

\section{La période de sensibilité tératogénique} modérée :

Elle couvre la période fœtale (de la 8 e à la $38 \mathrm{e}$ semaine).

Les anomalies déterminées durant cette période constituent des fœtopathies.

Les facteurs tératogènes agissent durant la période foetale et ne perturbent que la fonction. Ils sont qualifiés de facteurs physio-tératogènes, par opposition aux facteurs morphotératogènes qui induisent des anomalies de la forme.

Les anomalies déterminées durant la période fœtale sont très souvent viables, plus que les embryopathies.

Les malformations maxillo-faciales, à savoir les fentes orales, les bourgeons hypoplasiques et dysplasiques, les langues bifides détectées chez les souris de nos expériences sont déterminées durant la période fœtale. Ces malformations correspondent à des fœtopathies.

Les hypoplasies et les dysplasies des bourgeons dentaires sont dues à des défauts de prolifération cellulaire. Ce phénomène peut conduire à des agénésies totales ou partielles d'une ébauche embryonnaire [1, 2, 3].

Exemples :

- Agnathie : absence des maxillaires.

- Agénésie des bourgeons dentaires.

- Micrognathie et microphtalmie correspondent respectivement à des insuffisances de développement du maxillaire et de l'œil.

L'accolement de la langue au plancher buccal est dû à une absence de mort cellulaire physiologique. En effet, au cours du développement normal, de nombreuses cellules meurent par apoptose. Ces cellules sont devenues inutiles, voire indésirables. Elles doivent être sacrifiées pour permettre le modelage d'une structure, c'est-à-dire permettre à cette structure d'acquérir sa forme définitive. 
Les fentes orales sont la conséquence d'une absence ou insuffisance d'un mouvement morphogénétique. En effet, l'absence de soudure des processus palatins est à l'origine de fentes palatines et l'absence de soudure des bourgeons intermaxillaire et maxillaire est à l'origine des fentes labiales.

Les chiffres de I'OMS (1997) montrent que $12 \%$ de la population féminine mondiale fume. Ce taux s'élève à $24 \%$ dans les pays industrialisés [12]. Actuellement, un tiers des adolescentes fument et l'écart entre garçons et filles se resserre considérablement.

Cette progression a des conséquences directes sur le tabagisme maternel : on considère qu'un quart des femmes enceintes fument au cours de leur grossesse.

L'analyse des données bibliographiques $[9,10]$ montre d'une part, les effets qui ont pu être décrits avec chaque composant du tabac et d'autre part, les types de malformations qui ont été à ce jour associés au tabagisme.

Fumer une cigarette libère plus de 4000 substances toxiques, qui se répartissent en une phase gazeuse et une phase particulaire[8]. La toxicité de chacune des substances dépend de la force d'inhalation, du type de tabac, des expositions individuelles associées.

\section{$>$ Le monoxyde de carbone}

Il est le principal toxique de la phase gazeuse. Son affinité pour l'hémoglobine est 200 fois supérieure à celle de l'oxygène, avec une demivie beaucoup plus longue chez le fœtus que chez la mère.

Il augmente la carboxyhémoglobine fœtale et par conséquent, favorise I'hypoxie maternofœtale.

En expérimentation animale, on observe un risque augmenté d'anomalies squelettiques, aggravé par la déprivation protéique.
En clinique humaine, sur la base de suivis de grossesses, le risque tératogène apparaît dans des cas d'intoxication grave et/ou chronique.

\section{> Le dioxyde de carbone}

II représente 7 à $10 \%$ de la phase gazeuse. Chez I'animal, des anomalies cardiaques et squelettiques ont été retrouvées pour des doses correspondant à celles rencontrées habituellement dans l'environnement.

Il n'existe pas d'études, à notre connaissance, sur l'exposition isolée de la femme enceinte.

\section{$>$ La nicotine}

La nicotine est un composant de la phase particulaire. C'est la substance pour laquelle nous disposons de plus d'informations. La nicotine est vasoconstrictrice sur les vaisseaux utéroplacentaires et altère les échanges placentaires. Elle est aussi responsable d'une hypoxie fœtale.

L'augmentation de sa perméabilité placentaire serait plus importante en début qu'en fin de grossesse.

Chez l'animal, on a mis en évidence une augmentation des fentes orales et des anomalies squelettiques.

En clinique humaine, l'effet délétère observé est une augmentation modérée des avortements spontanées.

\section{$>$ Le cyanide}

Parmi les autres toxiques présents en phase particulaire, le cyanide pourrait avoir des conséquences sur l'organogenèse. En effet, lors de sa détoxification en thiocyanate, il mobilise la vitamine B12 et les acides aminés qui ne sont donc plus disponibles pour la synthèse protéique de l'embryon. 


\section{$>$ Le cadmium}

Le cadmium est retrouvé dans les ovaires, le liquide folliculaire et le placenta.

Sur le placenta il agit en se fixant sur les récepteurs du zinc par un mécanisme compétitif, et par conséquent altère la fonction placentaire.

\section{$>$ Le plomb}

Le plomb est reconnu comme une molécule neurotoxique. II traverse le placenta et à forte dose, constitue un facteur de risque d'avortement.

\section{> Les hydrocarbures aromatiques et le nickel}

Ce sont deux substances considérées comme étant tératogènes chez l'animal, mais il n'y a pas de données précises en clinique humaine.

Des différents effets décrits avec les composants, c'est le phénomène d'hypoxie maternofœtale qui est à retenir, principalement observé avec le monoxyde de carbone, le dioxyde de carbone et la nicotine. Chez l'animal soumis à des conditions d'hypoxie, on retrouve une augmentation des anomalies squelettiques, des fentes orales et des craniosténoses.

En clinique humaine, la survenue d'angiomes placentaires a été observée chez des femmes enceintes vivant à haute altitude.

Parmi les malformations retrouvées chez les fœtus de notre expérience, curieusement aucune fente palatine isolée $n$ 'a été découverte, nous avons remarqué un élargissement des fentes labio-palatines sur 4 des 33 embryons de souris étudiés. Les fentes orales ont fait l'objet de nombreuses études[13, 14] dont les conclusions sont variables.

Concernant la prévalence des fentes labiopalatines, le nombre d'individus atteints est de :
- 2-3/1000 chez les asiatiques ;

$-1 / 1000$ chez les caucasiens ;

$-0,4 / 1000$ chez les africains.

Cette variation selon la race montre que la génétique est impliquée dans la genèse de la fente.

Les recherches actuelles s'orientent vers une interaction entre gènes de prédisposition et tabac.

Toutes les études s'accordent à reconnaître que le tabac est un facteur à risque non négligeable pour la genèse des fentes. Elles s'appuient sur des méthodologies différentes, avec des échantillons variables mais aboutissent, pourtant, aux mêmes conclusions. Ceci est vrai pour les fentes labio-palatines, mais pas pour les fentes palatines qui ne semblent pas liées à un tabagisme maternel en début de grossesse. La fente est-elle une malformation d'apparition récente dans la race humaine et provoquée par la pollution?

La réponse est non et elle est illustrée par cette bouteille sculptée se trouvant dans un musée au Pérou et réalisée il y a plus de 1500 ans. Elle représente la tête d'un jeune homme porteur d'une fente labiale (fig. 16).

Un axe de recherche a été développé depuis plusieurs années sur les interactions génétique/environnement, notamment sur les interactions entre la consommation tabagique en début de grossesse et certains gènes de prédisposition[14].

Il semble exister une potentialisation du risque lorsqu'une femme est exposée au tabac et qu'elle est porteuse d'un allèle rare du gène TGF alpha.

Ceci n'est pas retrouvé dans toutes les études du fait des différences de susceptibilité qui existent d'une population à l'autre. Cette potentialisation relative du risque est également retrouvée pour les gènes TGF b3 et MSX1. 


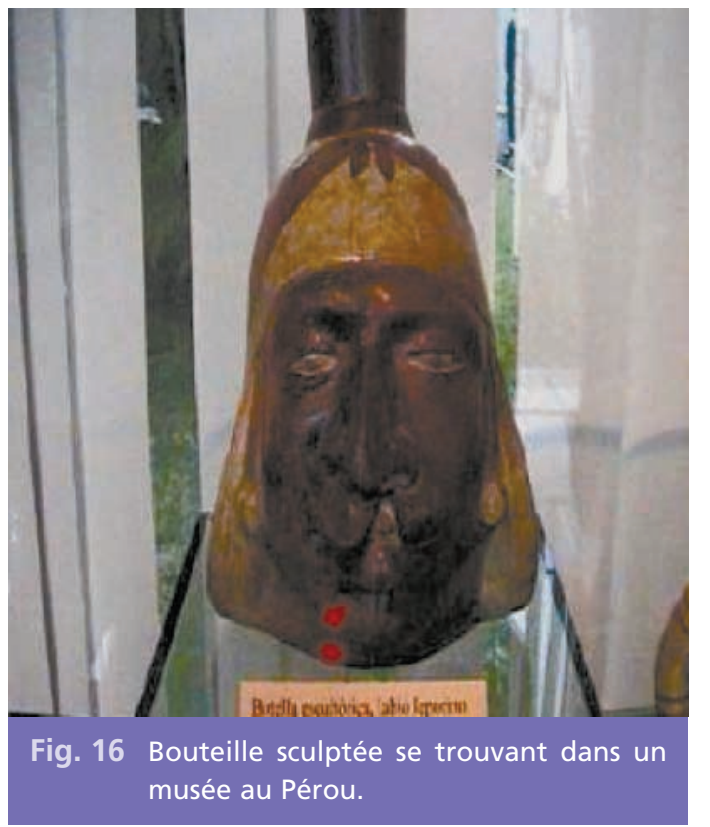

\section{Conclusion}

La revue de la littérature ne rapporte pas de relation entre tabac et malformations maxillofaciales autre que les fentes orales. Cependant notre travail a révélé l'apparition de plusieurs autres malformations telles que les kystes folliculaires, les dysplasies et hypoplasies des bourgeons dentaires et les accolements de la langue au plancher buccal.

Les résultats de nos expériences ont montré que $48 \%$ des embryons de souris exposés au
D'autres études ont été menées sur l'interaction entre le tabac et certains gènes détoxifiants, capables de protéger la cellule contre les composants néfastes de la fumée de tabac. Elles ont montré une potentialisation du risque lorsque les mères étaient porteuses d'un allèle non fonctionnel. Ce risque est accru lorsque le fœetus est également porteur d'un gène non fonctionnel car ces gènes détoxifiants agissent dans la période fœtale.

L'étude de l'interaction entre les facteurs génétiques et les facteurs environnementaux est une piste très intéressante exploitée depuis une petite dizaine d'années, mais les résultats sont assez modestes. Cette piste sera certainement développée dans l'avenir.

tabac sous toutes ses formes présentent une malformation maxillo-faciale.

Finalement, même s'il n'existe pas de preuves formelles encore suffisantes pour affirmer l'effet tératogène du tabagisme parental, les arguments sont nombreux en sa faveur et pourraient expliquer la fréquence des malformations survenant dans la population générale[15].

\section{Bibliographie}

1. Attité-Bitach $\mathrm{T}$, Vekemans $\mathrm{M}$, Encha-Razavi $F$.

Développement craniofacial : morphogenèse et déterminisme. Arch Pédiatr 2001, 8 Suppl 2:390-392.

2. Cohen MM, Gorlin FJ, Fraser FC.
Craniofacial Disorders.

Emery and

Rimoin's principles and

Practice of Medical Genetics, 3e édition. New York; 
Churchill Livingstone, 1997;

1121-1147.

3. Couly G.

\section{Malformations}

de la face

et du cou.

EMC 1992, 22066A10, 1-12.

4. Czyba JC, Dumas $P$.

Développement

embryologique

de la bouche et de la face.

EMC 2003, 22005C10, 21-30.

5. De Santis M, Straface $G$,

Carducci B, Cavaliere AF,

De Santis L, Lucchese A.

Risk of drug-induced

congenital defects.

Eur J Obstet \& Gynecol

Reproduct Biol 2004;

117:10-19.

6. Ellison L.F,

Morrison $\mathrm{HI}$ et coll.

Les conséquences

du tabagisme

sur la santé

des fumeurs canadiens :

Mise à jour.

Santé Canada, Volume 20,

No 1,1999.

7. Gorlin RJ, Cohen MM,

Levin LS.

Orofacial clefting

syndromes:

general aspects.

Syndromes of the Head and Neck, $3 e$ édition.

New York:

Oxford University Press,

1990 ; chapitre 20:693-700.

8. Hoffman $D$

and Wynder EL.

Chemical constituents

and bioactivity

of tobacco smoke.

Tobacco:

A Major international

Health Hazard

(Zaridze $D$ et Peto $F$, eds).

Publication Scientifique

du Centre International

de Recherche sur le Cancer.

Volume 74, Lyon,

France, 1986.

9. Langman J,

Sadler TW.

Embryologie médicale :

développement humain,

normal et pathologique.

Ed, Masson,

Paris Milan Barcelone

1994;131-146,461.

10. Mc Donald $A D$,

Armstrong BG,

Sloan $\mathrm{M}$.

Cigarette

and coffee consumption

and prematurity.

Am J Public Health

1992;82:87-90.

11. Moore KL, Persaud TVN. The Developing

\section{Human.}

Clinically Oriented

Embryology,

6e edition.

Toronto;

W.B. Saunders Company

1998:245-252.

12. Organisation mondiale

de la Santé.

Tabac Alerte.

Journée mondiale

sans tabac, Genève, 1996.

13. Precious DS, Goodday RH,

Morrison AD, Davis BR.

Cleft Lip and Palate:

A Review for Dentists.

Journal

of the Canadian

Dental Association

2001, vol, 67, n 11, 668-673.

14. Shaw $\mathrm{GM}$, Wasserman $\mathrm{CR}$, Lammer EJ et coll.

Orofacial clefts,

parental cigarette

smoking,

and transforming

growth factor-alpha

gene variants.

Am J Hum Genet 1996;58:

551-556.

15. Sohan $\mathrm{K}$, Freer $\mathrm{M}$,

Mercer $\mathrm{N}$ et coll.

Prenatal detection

of facial clefts.

Fetal Diagn Ther 2001;

4:196-199. 


\section{SUMMARY}

\section{Teratogenic effects of tobacco}

\section{on the mice orofacial structures}

Souha BENYOUSSEF BOUDEGGA

Badreddine SRIHA

Lamia OUALHA

Aicha ZAGHBANI

Fredj BENYOUSSEF

Chadly BACCOUCHE

Keywords

- teratology

- nicotinism

- maxillo-facial

malformation
The authors work consists in an experiment carried out on mice exposed to the different phases of tobacco during all the period of gestation in order to observe the anomalies of development concerning their embryos' maxillaries and faces. The results were alarming and significant which affirms the teratogenic effect of tobacco. 